\title{
Human Resource System of Colleges Based on Mobile Web of Technology
}

\author{
Ma Hongyan \\ Dept. of Organization and Human Resources \\ Qingdao Ocean Shipping Mariners College \\ Qingdao, China \\ E-mail: mahyan@163.com
}

\author{
Li Jianguo \\ The Library and Information Center \\ Qingdao Ocean Shipping Mariners College \\ Qingdao, China
}

\begin{abstract}
For the demand of mobile and convenient application for university personnel management system in the Internet era, this paper designs a set of personal management system of colleges based on mobile web technology. Using Sencha Touch of mobile web technology as programming tool, the system is created consist of the new staff enter management module, the staff unified management module, permission configuration module, the identity authentication management module, etc. then the mobile and intelligent management of university personnel management is realized. It not only can replace some daily recruit management tedious jobs but also can fully utilize Internet/Intranet to collect and issue information fast. The instance gives a good way to develop Web-Based Human Resource System.
\end{abstract}

Keywords-mobile web; Sencha Touch; personnel management system; system design

\section{INTRODUCTION}

The growing development of mobile web technology has put forward higher requirements to the Internet centered on TCP/IP technology, and along with the improvement of technology, it is bound to achieve mobile information control with TCP/IP as the core technology. Mobile web technology has the advantages of low development cost, multiple mobile devices suitability, cross-platform capability, easy iteration and update and many other advantages. How to play the better advantage of mobile web technology has become an important research branch in the field of computer and communications $[1,2,4]$. The new mobile Internet form essentially allows applications to through a browser, rather than through a specific operating system or a stationary operating platform. With the development of times, College Personnel Management System also puts forward new demands for traditional personnel management system.

In view of this situation, we design a University Personnel management system based on mobile web technology, the use of its mobility can easily access the data, remote control data read permissions, etc. and it has a good application in campus network, which has more obvious advantages than the traditional technical architecture personnel system in terms of technical architecture.

\section{RELEVANT BASIC KNOWLEDGE}

\section{A. Mobile Web Technology}

Mobile Web, one of the core technologies of the cloud. Internet era, it has strong advantages. It can be implemented in any mobile browser simply through XHTML, CSS and JavaScript. Many Web developers are turning to the construction of Web-based application framework [2], and its development tools are also increasingly rich. There are three main categories. The first category is Sencha Touch, which is the world's first HTML5-based Mobile App framework, with beautiful user interface components and rich data management, based on the latest HTML5 and CSS3 WEB standard, fully compatible with Android and iOS devices. Sencha Touch is a relatively heavyweight mobile Web application framework, suitable for the development of mobile applications with more complex business logic. It has pure JavaScript design layout with excellent version stability. The second category is jQuery Mobile, the version in mobile phones and tablet devices. It not only brings the jQuery core library to the mainstream mobile platform, but also releases a complete and unified jQuery mobile website based on HTML5, with the advantage of super multi-platform support. The third category is PhoneGap, a quick development platform based on HTML, CSS and JavaScript, to create mobile applications. It enables developers to take advantage of iPhone, Android, Palm, Symbian, WP8, Blackberry and other smart phone's core functions and has extensive plugin. Its defect is slow running and delayed UI response.

\section{B. Brief Introduction of Sencha Touch}

According to the above analysis and the characteristics of Universities Personnel Management System, this paper chooses cross-platform, rich interactive Sencha Touch as a programming tool.

Sencha Touch uses a three-tier structure model, that is, customer layer, business logic layer (model) and the server layer. Among them, the client layer is to provide users with interactive operation interface and by calling the business logic layer to indirectly operate the background database; Business logic layer, the link between the upper and lower layers, is to achieve the user's request, and eventually return the corresponding results to the customer level; The server layer is 
the bottom of the three-tier structure with data storage and retrieval as its assignment. This structure model has a certain degree of flexibility. When the local business logic changes, it will not have an impact on other business components, and the system can be developed separately. Developers can flexibly choose the programming language to achieve the development of components and decompose complex functions of application system into many small modules or rely on the support provided by the system, it has strong stability and reliability [3].

The application includes a collection of Model, View, Controller, Store, and additional metadata, such as icon icons and images that are displayed on the launch interface. Model can achieve business logic management, representing the object of management in the, and it is used through the model manager registration and the store data binding of ext. View is to display data to users who can have a look at the system interface. The controller listens on events triggered by the UI interface and makes the corresponding operations to isolate the interface logic from the control logic. Each controller of the application is a subclass of ext.app.controller, or it can inherit an existing controller. The store is responsible for loading the data into the application and displaying it in the form of a list or a data view. Profiles are used to customize the application user interface for tablet or phone, and share program code as much as possible. Profiles are simple classes that define the different types of devices that the program supports and how to handle the differences between them. Profiles are not necessary which can be added later or never be defined. Each profile must define a simple isActive function, and if the current profile starts, it will return true, while loading the corresponding profile models, views and controllers. Sencha Touch application's object model is shown in Figure 1.

\begin{tabular}{|c|c|}
\hline \multicolumn{2}{|c|}{ presentation layer } \\
\hline \multicolumn{1}{|c|}{ Data Access Layer (obtain local data } \\
through Ajax $\backslash$ Jsonp $\backslash$ Yql etc. web socket )
\end{tabular}

Fig. 1. Sencha Touch Object Model

\section{SYSTEM DESIGN PRINCIPLE}

The design principles of the system are as follows:

1) Functional modules. It should as far as possible contain the departments involved in the business, such as employee registration management, unified management of staff data, access to different users, etc. The work process needs to be scientific and reasonable, so as to improve the services of the sector effectiveness.

2) Mobility of system data access. This is the characteristics of the system, the mobile devices used by the new staff, teachers and managers, such as mobile notebooks, mobile phones, PDA, etc. can complete the relevant data operation, and the interface is to be friendly and convenient.

3) The security of system data. In order to protect the system from human damage, users need to be authenticated to access the database, which is the system to focus on the issue.

4) System compatibility and interoperability. We should take into account data compatibility and exchange between this system and relevant departments, school education system, scientific research management system, library system, financial system, asset system, office system, logistics management system, journal system and other units[5-6].

\section{SySTEM DESIGN MODULE}

In this paper, the design is divided into four modules, namely, enrolling management of new employee, staff unified data management, configuration management authority, identity authentication management.

\section{A. New staff management}

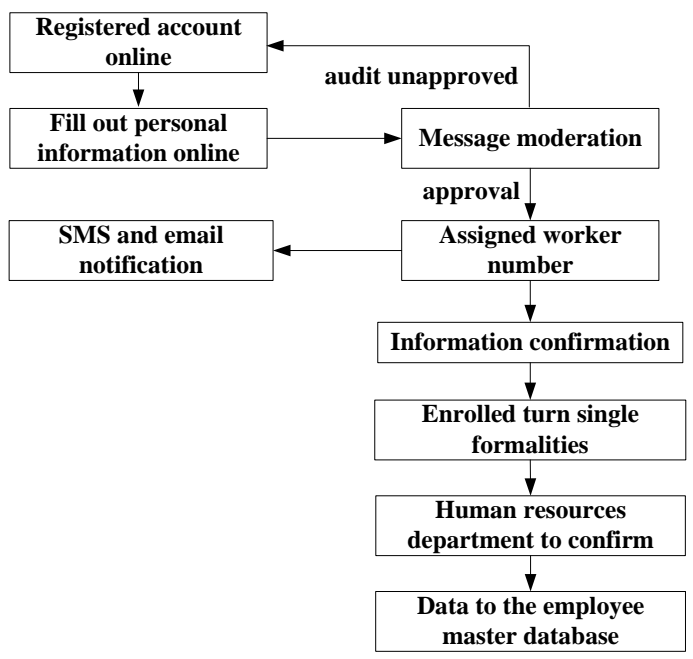

Fig. 2. New employee enrolled process

Staff registration management is an important process of personnel system, mainly to complete the collection of basic information of the staff. It is designed from the school's overall business, forming a school-wide universal school registration center. The new staff will write their information, including: basic information, learning experience, work experience, family members. The information then will be submitted to the audit, after the first personnel audit, the system will generate job numbers and send SMS notification to the faculties through the SMS platform interface to go through the formalities of the new staff's enrollment into the school and organize the department of human resources to confirm the list. Their personal information then will be synchronized to the OneCard center to do camera preparation; after the live register of the new staff, the management department will update the starting salary date and other management information and send this notification to the pay office by calling the message center interface, the staff office, personnel file room and other departments to complete the corresponding information audit and operation; then the staff will complete the procedure into 
the school process, the system will extract the basic information to the shared library, distributing to the business system to complete the initialization operation of user's rights. New staff, managers use mobile devices, including mobile notebooks, mobile phones, PDA, etc., to complete the relevant data operation, as shown in Figure 2.

\section{B. Unified Management of Faculties}

Personnel master data, including the subject of proprietary data, external data and foreign data, will achieve unified management of the faculty data, so as to effectively realize the separation of service and management functions. The changes of management business will not affect the interface and its main function, maintaining user interface stability and unity. Subject proprietary data includes organizational structure, job information, retired personnel information, salary and welfare information, job information, contract information and so on. Personnel data and other data exchanges include businesses that need to be done, to be processed and to be approved etc.; business management notices that need cooperation among different departments; all kinds of reminders; system notifications. Faculty can use the mobile device to complete the relevant data operation, seeing in Figure 3

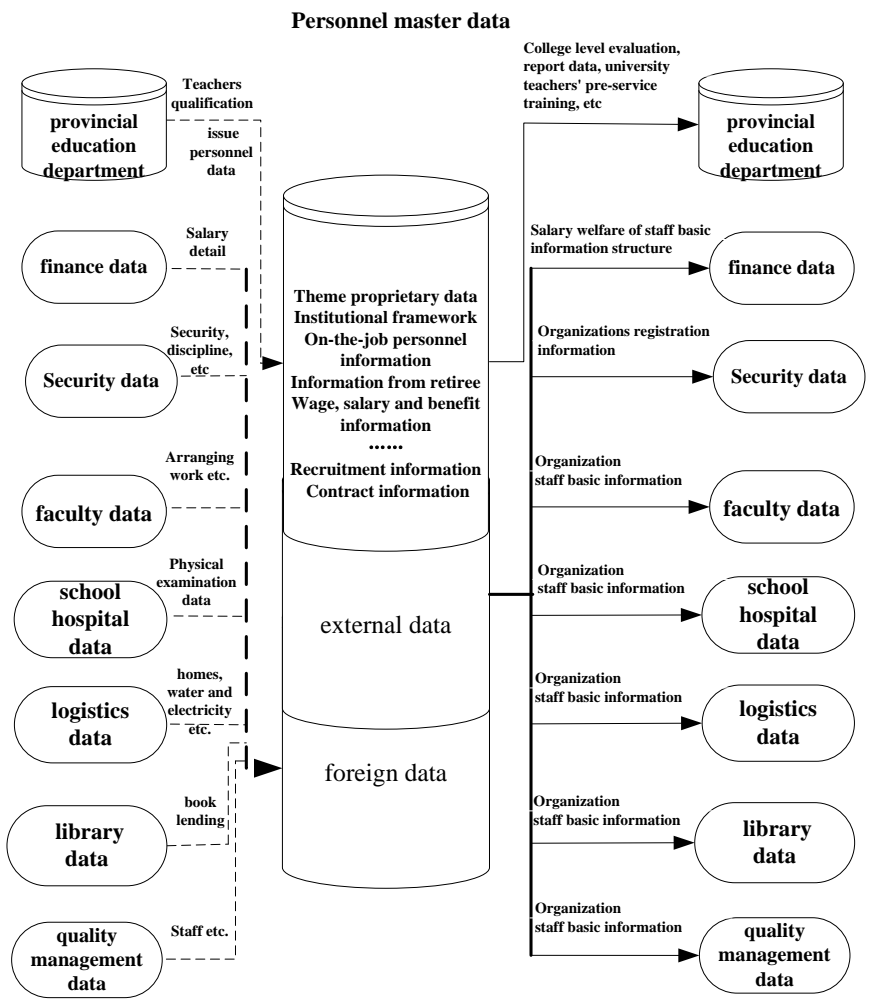

Fig. 3. Personnel data and other data exchange

The system builds a unified communication platform to realize the news integration, so as to effectively integrate the message resources in the university heterogeneous environment, solve the demand of data transmission in distributed system, and complete the school-wide message processing. All the business systems including the personnel management system need to comply with the message center's norms while constructing, organically integrate message resources, set out rules and sort messages, pushing attractive information in different ways (such as SMS, e-mail, RSS Subscription, etc.) to the customer service center. After the update of information source, users can accurately have access to personalized services in time.

\section{Rights Configuration Management}

The authority management of this system, based on the role-function-resource permissions control method, associates access and role which then associates with the user to achieve the logical separation between user and access, providing extended support for personalized services to the teachers and the students. It mainly includes the following data tables: the user table recording the user's information; role table recording the role of the relevant information; module and function table recording module related information and module related functions; resource table recording the resource information that needs high security Control.

Permission configuration management is divided into three parts. The first part is rights data maintenance which is responsible for maintaining some basic information, such as users, roles, functions, resources, etc., providing a convenient operation interface for the administrative staff of this system. The second part is authority data processing. This part is responsible for the implementation of the authority calling interface within the program, such as providing operational data and functions according to the caller and the user's information. The third part is authority data citing. The presentation layer performs a specific processing of the combination rights of the user, for example, to control the display or concealment of the function elements such as buttons, menus, and the like on the UI according to the obtained function authority information.

\section{Identity Authentication Management}

The system takes into account the safety. In order to protect the system from human damage, users need to be authenticated to access the database. The user needs to submit requests to the certificate server through SSL (Secure Sockets Layer Protocol), and the certificate is stored in the directory server (LDAP Server) which could provide users with the service to find user information, such as the user's IP address and the department information to realize user-friendly further query [7-9]. The process is as follows: Server authentication phase, where the server initiates a new session connection and sends the start message "hello" to the server; the server determines whether a new master key needs to be generated based on the information and, if necessary, the server returns a response message which contains the information needed to generate the master key; the client creates a new key according to the response information and encrypts it with the public key of the server and sends it to the server; the server responds to the master key, and the information authenticated by the master key is sent to the customer, and the customer authenticates the server with this information. User authentication phase: After the server is authenticated, the customer will be authenticated. The server that has been authenticated sends a question message to the client, and the client returns the (digital) signature and its public key to the server to provide authentication to the server. 


\section{CONCLUSION}

This paper designs the personnel management system of university based on mobile web technology, and has obvious advantages such as convenient and high security compared with the traditional technical system of personnel system. The system can effectively solve the personnel management of mobile convenience problems, but in dealing with more complex personnel relations, it is still insufficient and needs further improvement.

\section{REFERENCES}

[1] K.Zhang,Y.M.Yu, and Y.Mi, "Human Resource System of Colleges Based on One-Stop Service Model," Journal of Wuhan University(Natural Science Edition),2012,58(s1) 297-301

[2] Y.L.Zhu, "Design and Implementation of Rapid information Collection System Based on Mobile WEB Technology," Journal of Electric Power, 2013,02:152-155.
[3] http://docs.sencha.com/touch/2.2.1/

[4] Y.M.Wei, "Design and Implementation of Personnel Management System," Jilin Normal University Journal(Natural Science Edition),2012,04:126-128

[5] J.L.Zhang, C.H.Zhang, "University personnel management system design and implementation based on improved MVC," Computer Engineering, 2004,(8): 191-194.

[6] G.C.Weng, "On the Informatization of University Human Resources Management," Journal of Ningbo University(Educational Science Edition), 2010,01:79-82+101.

[7] C.F.Xing, Y.Li, and H.B.Zhao, etc., “A Mobile Web Service Security Technology Scheme," Computer Technology and Development, 2013,04:122-125

[8] L.Wu, "Information construction of human resource management in colleges and universities," Journal of Jiangxi University of Science and Technology,2009,02:68-70.

[9] M.X.Chen, "Problems and countermeasures of human resources information management," Market Modernization,2008,23:282. 